\title{
Improvement of sensory odour intensity scale using 1-butanol reference solutions for environmental odour evaluation
}

\author{
Higuchi T. ${ }^{*}$, Sekine M., Imai T., Yamamoto K. and Kanno A. \\ Graduate School of Sciences and Technology for Innovation, Yamaguchi University, 2-16-1, Tokiwadai, Ube, Yamaguchi 755-8611, Japan \\ Received: 30/05/2018, Accepted: 26/09/2018, Available online: 27/09/2018 \\ *to whom all correspondence should be addressed: e-mail: takaya@yamaguchi-u.ac.jp \\ https://doi.org/10.30955/gnj.002779
}

\begin{abstract}
For appropriate evaluation of environmental odours, it is necessary to develop a reliable odour measurement scale. Odour intensity reflects people's perception of odours and contributes to effective odour management. In this study, Japanese conventional six-point odour intensity scale was reconsidered to ensure equal intervals between odour intensity levels. A new series of six dilution steps of 1-butanol solutions with a concentration of $0,10,600$, 2600, 9000 and $22500 \mathrm{ppm}$ (vol/vol) was proposed and explanatory labels for odour intensity levels were determined. In addition, polyethylene (PE) bottles were proposed as containers for 1-butanol odour intensity reference solutions for convenience in on-site handling, and the effectiveness of PE bottles was suggested. These results showed that the new odour intensity scale would be applicable to on-site investigations and practically useful for all people related to environmental odour evaluation.
\end{abstract}

Keywords: Dilution step, explanatory label, on-site investigation, polyethylene bottle.

\section{Introduction}

Odours discharged from various human activities may cause severe damage to residents. For appropriate evaluation of environmental odours, it is necessary to develop a reliable odour measurement scale. Since environmental odours consist of a large variety of odorous compounds, comprehensive evaluation of odours using human sense of smell as well as instrumental analysis of individual chemicals is indispensable. Odour intensity is one of main odour characterization parameters (Naddeo et al., 2013) and remarkably common and important sensory indicator of environmental odours. Odour intensity reflects people's perception of odours and contributes to effective odour management. Several odour intensity scales have been developed and used for decades in the world (Cha, 1998; WEF, 2004; Naddeo et al., 2013).

In Japan, the six-point odour intensity scale shown in Table 1 was developed more than 40 years ago and the regulation standards based on the Offensive Odour Control Law were set equivalent to the odour intensity that ranges from 2.5 to 3.5 on this scale (Higuchi and Nishida, 1995). In the measurement, six or more panel members sniff a testing odour directly and classify their impressions in accordance with the scale in 0.5 segments. After discarding the maximum and the minimum values, the remaining values are averaged (Iwasaki, 2017). This scale is very easyto-use, acceptable to residents and applicable to any fields at any time. On the other hand, the independent judgments of the panel members are subjective and equal intervals between intensity levels are not necessarily ensured.

Table 1. Six-point odour intensity scale

\begin{tabular}{cc}
\hline Level & Odour intensity \\
\hline 0 & No odour \\
\hline 1 & Barely perceivable (Detection threshold) \\
\hline 2 & Faint but identifiable (Recognition threshold) \\
\hline 3 & Easily perceivable \\
\hline 4 & Strong \\
\hline 5 & Extremely strong \\
\hline
\end{tabular}

Some countries have developed their unique odour intensity scales including VDI 3882 Part 1 (VDI, 1992) in Germany and ASTM E544-10 (ASTM, 2010) in the U.S.A. According to VDI 3882 Part 1, odour intensity measurements are carried out with dynamically diluting olfactometers. The category scale of odour intensity is primarily an ordinal number scale and a specified ranking is assigned to its categories. ASTM E544-10 describes dynamic and static scales both designed to compare the odour intensity of the sample with the odour intensities of a series of 1-butanol concentrations. In the dynamic scale method, a dynamic-dilution apparatus is used and vapor dilutions are prepared by continuous mixing of vapors of 1 butanol and odourless air. In the static scale method, dilutions of 1-butanol in water are prepared in Erlenmeyer flasks and presented for odour intensity comparison. At least eight independent judgments of the panel members are obtained and averaged geometrically with respect to the 1-butanol concentrations of the matching points. A geometric progression scale with a ratio of two is recommended and odour intensity levels are clearly defined in both methods. 
Considering principles and ideas of several odour intensity scales, above-mentioned disadvantages of Japanese sixpoint odour intensity scale could be improved. In this study, Japanese conventional six-point odour intensity scale was reconsidered and a new series of six dilution steps of 1-butanol and explanatory labels were proposed to ensure equal intervals between odour intensity levels with reference to the static scale of ASTM E544-10. In addition, polyethylene (PE) bottles were proposed instead of Erlenmeyer flasks as containers for 1-butanol odour intensity reference solutions for convenience in on-site handling.

\section{Materials and methods}

\subsection{Determination of 1-butanol dilution steps}

First, 1-butanol dilution steps in which adjacent odour intensity could be discriminated were determined. Two wide-mouth Erlenmeyer flasks with a capacity of $500 \mathrm{~mL}$ were prepared and adjacent reference solutions of 1-butanol (CAS registry number: 71-36-3) were placed into them. The volume of solution was $200 \mathrm{~mL}$ and the top of each flask was covered with aluminum foil between sniffing. The panel members gently shook flasks prior to each sniffing to ensure equilibrium and odour intensity was evaluated by the magnitude estimation method. Experiments were conducted using 1-butanol dilution steps with a ratio of 3, 4, 5.3 and 5.6. Experimental results were evaluated using Wilcoxon signed rank test. A total of 20 panel members who ranged from 21 to 24 years of age and passed Japanese panel screening test (JEA, 1995) joined the experiment.

Second, 1-butanol dilution steps that ensure equal intervals between odour intensity levels were determined. Seven groups of reference solutions with different dilution steps were prepared and presented to the panel members. The panel members sniffed each flask and evaluated perceived odour intensity by placing a magnetic strip on a line segment with a length of $250 \mathrm{~mm}$ on a whiteboard as shown in Figure 1 (Takemura et al., 2009). Magnetic strips labelled $\mathrm{A}$ to $\mathrm{F}$ corresponded to 1-butanol dilution steps. After the evaluation, the distance between magnetic strips was measured. A total of 30 panel members who ranged from 21 to 24 years of age and passed Japanese panel screening test (JEA, 1995) joined the experiment.

\subsection{Determination of explanatory labels for 1-butanol dilution steps}

Appropriate explanatory labels for 1-butanol dilution steps proposed in 2.1 were determined. Wide-mouth Erlenmeyer flasks with a capacity of $500 \mathrm{~mL}$ were prepared and reference solutions of 1-butanol were placed into them. The volume of solution was $200 \mathrm{~mL}$. The panel members sniffed each flask and chose the most appropriate explanatory label from 13 choices on the evaluation sheet. Choices were selected from the conventional six-point odour intensity scale and papers related to the sensory evaluation of odour and noise. A total of 18 panel members who ranged from 21 to 24 years of age and passed Japanese panel screening test (JEA, 1995) joined the experiment.

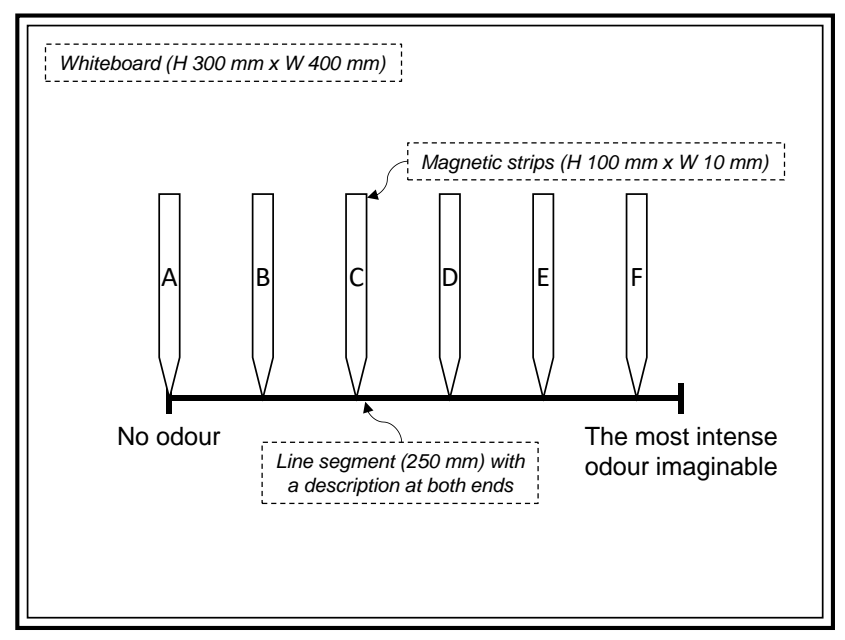

Figure 1. Odour intensity evaluation using a whiteboard and magnetic strips

After the determination of explanatory labels, the validity of the new odour intensity scale was confirmed by odour intensity measurement tests using 1-butanol as quasi environmental odour. Odour intensity of three samples with known odour intensity was measured and consistency of experimental results was investigated. A total of 50 panel members who ranged from 21 to 24 years of age and passed Japanese panel screening test (JEA, 1995) joined the experiment.

\subsection{Comparative odour intensity measurement using Erlenmeyer flasks and PE bottles}

Wide-mouth Erlenmeyer flasks with a capacity of $500 \mathrm{~mL}$ are breakable and bulky in on-site handling. To improve these problems, PE bottles with a capacity of $400 \mathrm{~mL}$ were selected as new containers for 1-butanol odour intensity reference solutions. PE bottles fulfill essential conditions as follows:

1. They have no odour,

2. No odour is adsorbed on their surface,

3. They are inexpensive and easily obtained,

4. Diameter of the bottle mouth $(40 \mathrm{~mm})$ is as same as that of Erlenmeyer flask (47 mm), and

5. They are reusable after cleansing with odourless detergent.

Photo 1 shows a PE bottle.

Odour intensity measurement was carried out to investigate whether PE bottles could be substituted for Erlenmeyer flasks as containers for 1-butanol reference solutions. Six wide-mouth Erlenmeyer flasks with a capacity of $500 \mathrm{~mL}$ were prepared and reference solutions of 1 butanol determined in 2.1 were placed into them. The volume of each solution was $200 \mathrm{~mL}$ and the top of the flask was covered with aluminum foil. The panel members gently shook flasks to ensure equilibrium, opened the covers and sniffed one by one to memorize odour intensity impressions. After taking a break of 3 minutes, the panel 
members sniffed 1-butanol sample solutions in PE bottles and judged odour intensity according to the 1-butanol reference scale. Three sample solutions with a concentration of 10, 600 and 2600 ppm (vol/vol) were presented. A total of 20 panel members who ranged from 21 to 24 years of age and passed Japanese panel screening test (JEA, 1995) joined the experiment. Odour intensity was evaluated 5 times repeatedly for each sample.

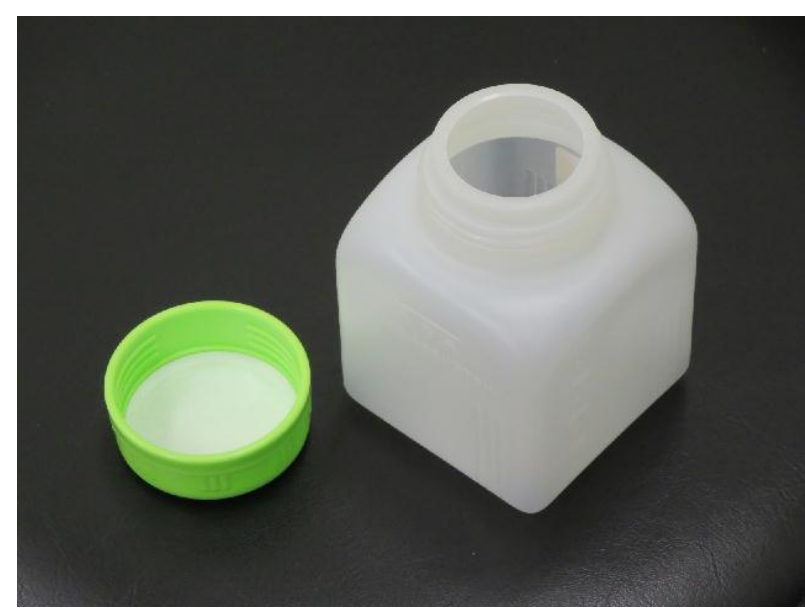

Photo 1. Polyethylene (PE) bottle

\subsection{Odour intensity measurement of hydrogen sulfide}

Odour intensity of hydrogen sulfide was measured to investigate the applicability of PE bottles for the evaluation of typical odorous substance. Six PE bottles were prepared and reference solutions of 1-butanol determined in 2.1 were placed into them. The volume of each solution was $200 \mathrm{~mL}$ and the top of the bottle was tightly capped. The panel members gently shook bottles to ensure equilibrium, opened the caps and sniffed one by one to memorize odour intensity impressions. After taking a break of 3 minutes, the panel members sniffed diluted hydrogen sulfide (CAS registry number: 7783-06-4) in polyethylene terephthalate bags and judged odour intensity according to the 1-butanol reference scale. Four hydrogen sulfide samples with a concentration of $0.006,0.02,0.06$ and $0.2 \mathrm{ppm}$ were presented. A total of 20 panel members who ranged from 21 to 24 years of age and passed Japanese panel screening test (JEA, 1995) joined the experiment. Odour intensity was evaluated 5 times repeatedly for each sample.

\section{Results and discussion}

\subsection{Determination of 1-butanol dilution steps}

Experimental results of odour intensity estimation at dilution steps with a ratio of 3 and 4 showed no statistical significant difference between adjacent reference solutions, suggesting that adjacent dilution steps could be hardly discriminated with a ratio of 3 and 4 . On the other hand, odour intensities of adjacent reference solutions with a ratio of 5.3 and 5.6 showed statistical significant difference (significance probability $p<0.05$ ). These findings imply that adjacent odour intensity of 1-butanol dilution steps with a ratio of at least 5.3 can be discriminated.

After the evaluation of seven groups of reference solutions, 1-butanol dilution steps that ensure almost equal intervals between odour intensity levels were determined as shown in Figure 2. These dilution steps consist of 1-butanol solutions with a concentration of $0,10,600,2600,9000$ and $22500 \mathrm{ppm}$ ( $\mathrm{vol} / \mathrm{vol}$ ). The coefficient of determination of the regression line was 0.999 and standard deviations were smaller in lower ranges and larger in higher ranges.

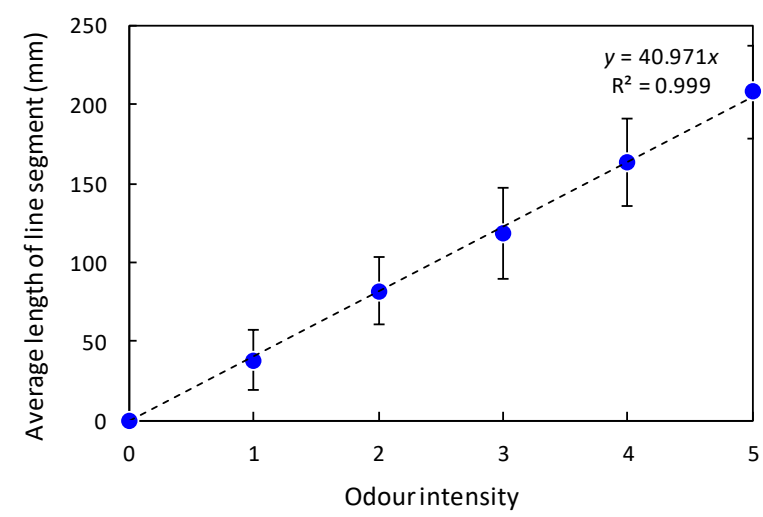

Figure 2. Relationships between odour intensity (1-butanol dilution steps with a concentration of $0,10,600,2600,9000$ and $22500 \mathrm{ppm}(\mathrm{vol} / \mathrm{vol}))$ and average length of line segment. Bars and a dashed line represent standard deviations and the regression line, respectively

\subsection{Determination of explanatory labels for 1-butanol dilution steps}

Since the panel members showed wide discrepancies in the explanatory labels, it was impossible to determine appropriate labels directly. The explanatory label that was not a general expression, covered multiple odour intensity levels and caused a wide variation among individuals was considered to be inappropriate and discarded from the choices. After the reconsideration of the choices, experiments for the determination of explanatory labels were conducted again using the remaining 9 choices. Statistical tests based on the binomial distribution were applied to investigate the significance of explanatory labels (Yamazaki et al., 2013). As a result, explanatory labels for odour intensity levels were determined as shown in Table 2.

The validity of the new odour intensity scale was confirmed by odour intensity measurement tests. Odour intensity of three samples which corresponded to reference solutions of level 1, 3 and 5 on the new scale was measured with increments of 0.5 . As a result, correct intensity values were significantly chosen by the panel members as shown in Figure $3(p<0.01)$. These results suggest that the proposed odour intensity scale can be practically useful without concern about olfactory adaptation and loss of memory.

\subsection{Comparative odour intensity measurement using Erlenmeyer flasks and PE bottles}

Three 1-butanol sample solutions with a concentration of 10, 600 and $2600 \mathrm{ppm}$ ( $\mathrm{vol} / \mathrm{vol})$, which corresponded to odour intensity level of 1,2 and 3, respectively, were presented in PE bottles. Odour intensity of these sample solutions was measured with increments of 0.5 . Figure 4 
depicts the results. Mean odour intensities of five repetitions were $0.9,2.3$ and 3.1 for sample solutions of intensity level 1, 2 and 3, respectively. Mean odour intensities at fifth repetition were $0.8,2.3$ and 3.1. The percentages of panel members who replied correct odour intensity at fifth repetition were $30 \%, 40 \%$ and $40 \%$ for sample solutions of intensity level 1, 2 and 3, respectively. These results suggest that PE bottles can be substituted for Erlenmeyer flasks as containers for 1-butanol odour intensity reference solutions, especially in the middle intensity level. Lower percentage of correct reply at low intensity level might be caused by indistinct odour perception.

Table 2. Six-point 1-butanol odour intensity referencing scale

\begin{tabular}{ccc}
\hline Level & $\begin{array}{c}\text { 1-Butanol concentration in } \\
\text { water }(\mathbf{p p m} \text { (vol/vol)) }\end{array}$ & Odour intensity \\
\hline 0 & 0 & No odour \\
\hline 1 & 10 & Faint \\
\hline 2 & 600 & Easily perceivable \\
\hline 3 & 2600 & Slightly strong \\
\hline 4 & 9000 & Strong \\
\hline 5 & 22500 & Very strong \\
\hline
\end{tabular}

$\square 10$ ppm (Intensity level 1 )

$\square 2600$ ppm (Intensity level 3)

$\square 22500$ ppm (Intensity level 5)

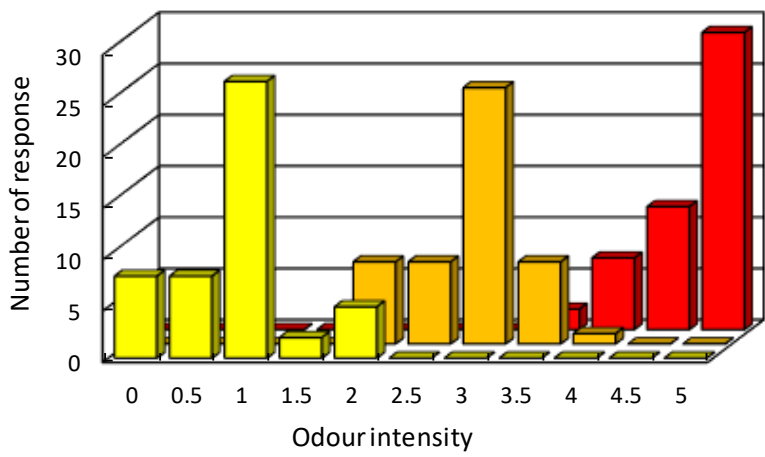

Figure 3. The number of response to three 1-butanol solutions

Mean standard deviations of five repetitions were 0.68 , 0.77 and 0.62 for sample solutions of intensity level 1, 2 and 3 , respectively. Standard deviations at fifth repetition were, however, $0.57,0.64$ and 0.55 . These results imply that the variation in odour intensity evaluation tend to be reduced with the increase of repetition.

\subsection{Odour intensity measurement of hydrogen sulfide}

Figure 5 shows odour intensities of four hydrogen sulfide samples. Mean odour intensities at fifth repetition were 1.6, 2.1, 3.0 and 3.5 for samples with a concentration of $0.006,0.02,0.06$ and $0.2 \mathrm{ppm}$, respectively. Standard errors at fifth repetition were $0.25,0.25,0.26$ and 0.19 . The variation of mean odour intensity over five repetitions was greater than that of 1-butanol solutions shown in Figure 4. These results suggest that, when 1-butanol solutions are used as reference odours, odour intensity evaluation of different odorous substances is accompanied by greater variation. Mean odour intensities are, however, relatively discriminable.
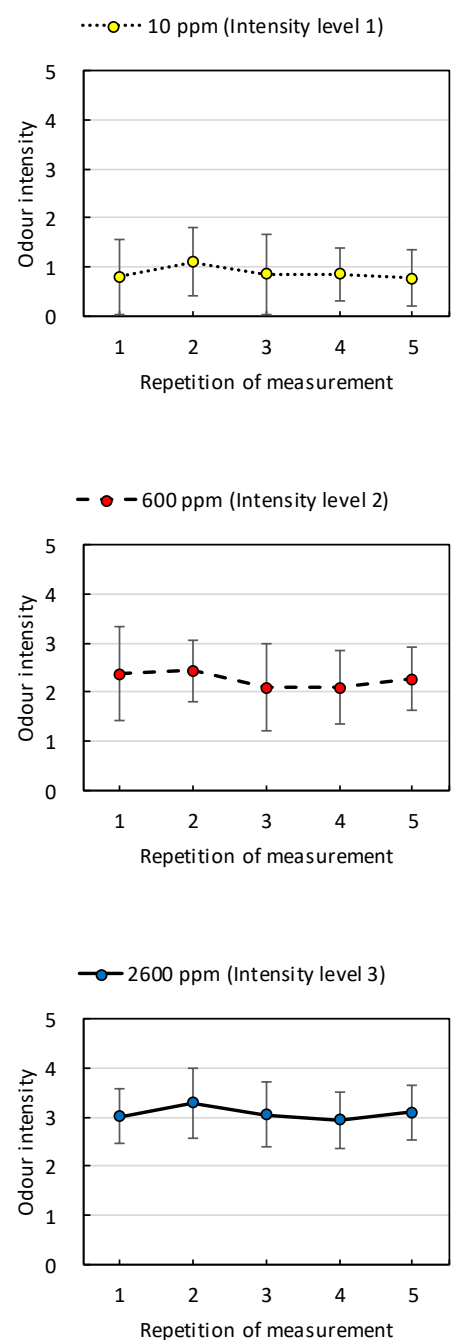

Figure 4. Odour intensities of three 1-butanol sample solutions in PE bottles. Circles and bars represent mean values and standard deviations, respectively

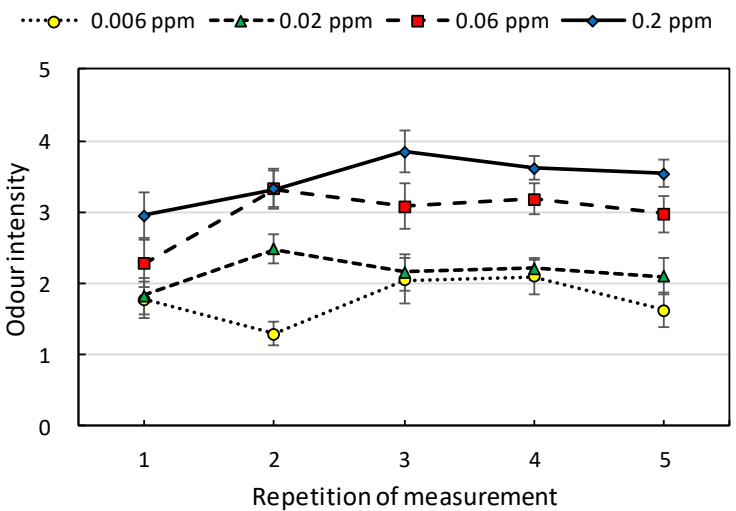

Figure 5. Odour intensities of four hydrogen sulfide samples. Marks and bars represent mean values and standard errors, respectively

\section{Conclusions}

In this study, Japanese conventional six-point odour intensity scale was reconsidered and a new series of six 
dilution steps of 1-butanol solutions with a concentration of $0,10,600,2600,9000$ and 22500 ppm (vol/vol) was proposed. Then, explanatory labels for odour intensity levels were determined. In addition, PE bottles were selected as containers for 1-butanol odour intensity reference solutions for convenience in on-site handling, and the effectiveness of PE bottles was suggested. The new scale will be applicable to on-site investigations and practically useful for all people related to the environmental odour evaluation. A comparative investigation on the reliability of odour intensity measurement between the method proposed in this study and other methods adopted in the world would be necessary in future.

\section{References}

ASTM E544-10 (2010), Standard Practices for Referencing Suprathreshold Odor Intensity.

Cha S.S. (1998), Sensory test methods, In: Odor and VOC Control Handbook, Rafson H.J. (Ed.), McGraw-Hill, New York, USA, pp. 4.16-4.32.

Higuchi T. and Nishida K. (1995), Analysis of data measured by the triangular odor bag method, Proceedings of the A\&WMA's International Specialty Conference, Odors: Indoor and Environmental Air, pp. 181-192.

Iwasaki Y. (2017), Olfactory Measurement of Odors, Japan Association on Odor Environment, Tokyo, Japan (in Japanese). Japan Environment Agency (1995), Determination of odor index and odor emission rate, Notification 63 (in Japanese).

Naddeo V., Belgiorno V. and Zarra T. (2013), Odour characterization and exposure effects, In: Odour Impact Assessment Handbook, Belgiorno V., Naddeo V. and Zarra T. (Eds.), John Wiley \& Sons, Chichester, UK, pp. 7-29.

Takemura A., Sagara K., Yamanaka T., Kotani H. and Fujimoto T. (2009), Comparison of psychological distance of words used for evaluation scale of olfaction, Journal of Environmental Engineering, Transactions of Architectural Institute of Japan, 74, 495-500 (in Japanese).

VDI 3882 Part 1 (1992), Olfactometry: Determination of Odour Intensity.

Water Environment Federation (2004), Odor sampling and measurements, In: Control of Odors and Emissions from Wastewater Treatment Plants, Water Environment Federation, Alexandria, USA, pp. 3-32.

Yamazaki K., Higuchi T. and Park S. (2013), Development of rating words suitable for odor intensity scale using 1-butanol, Proceedings of the 26th Annual Conference on Odor Environment, Japan Association on Odor Environment, pp. 5255 (in Japanese). 